\title{
Robotic Single-Site Hysterectomy versus Robot-Assisted Multiport Hysterectomy in Benign Gynecologic Diseases: A Retrospective Comparison of Clinical and Surgical Outcomes
}

\author{
Ye Hyon Park', Se Hee Park², Ryeo Bin Lee', Hye-Yon Cho', Jung Bae Kang', Pong Rheem Jang', Min Sun Kyung' \\ ${ }^{1}$ Department of Obstetrics and Gynecology, Hallym University Dongtan Sacred Heart Hospital, Hwaseong; ${ }^{2}$ Department of Obstetrics and Gynecology, Hallym \\ University Kangnam Sacred Heart Hospital, Seoul, Korea
}

\begin{abstract}
Objective: The purpose of this study was to compare clinical and surgical outcomes of robotic single-site hysterectomy (RSSH) and robot-assisted multiport hysterectomy $(\mathrm{RH})$ in benign disease.

Methods: We retrospectively reviewed the medical records of 38 women who underwent RSSH $(\mathrm{N}=12)$ or $\mathrm{RH}(\mathrm{N}=26)$ for the treatment of benign uterine disease between June 2015 and November 2017.

Results: There were no intergroup differences in parity, comorbidities, and number of previous abdominal surgery. Mean age was older ( $49.5 \pm 5.05$ years vs. $44.4 \pm 3.54$ years, $P=0.001)$ and mean body mass index was higher $\left(27.4 \pm 2.47 \mathrm{~kg} / \mathrm{m}^{2} \mathrm{vs} .25 .3 \pm 3.12 \mathrm{~kg} /\right.$ $\left.\mathrm{m}^{2}, \mathrm{P}=0.045\right)$ in RSSH group than RH group. Surgical outcomes, including operative time (165.0 minutes vs. 159.2 minutes, $P=0.727)$, estimated blood loss (115.8 $\pm 33.15 \mathrm{~mL}$ vs. $108.1 \pm 56.42 \mathrm{~mL}, P=0.662)$, uterus weight $(445.9 \pm 157.21 \mathrm{~g}$ vs. $374.5 \pm 197.91$ g, $P=0.291)$, postoperative hospital stay $(5.4 \pm 0.51$ days vs. $5.8 \pm 1.20$ days, $P=0.289)$, postoperative hemoglobin change in day 1 $(1.8 \pm 0.89 \mathrm{~g} / \mathrm{dL}$ vs. $1.4 \pm 1.53 \mathrm{~g} / \mathrm{dL}, \mathrm{P}=0.431)$ and day $3(2.1 \pm 1.32 \mathrm{~g} / \mathrm{dL}$ vs. $1.7 \pm 1.83 \mathrm{~g} / \mathrm{dL}, \mathrm{P}=0.601)$, and perioperative complications did not significantly differ between two groups. The use of additional analgesics after 6 hours, 24 hours, and 48 hours, and mean NRS score after 6 hours, 24 hours, and 48 hours were not significantly different between two groups.

Conclusion: RSSH might be an effective and safe alternative to $\mathrm{RH}$, even if in older and/or obese women with large uteri.
\end{abstract}

Keywords: Uterus; Hysterectomy; Minimally invasive surgical procedures; Robotic surgical procedures

\section{INTRODUCTION}

Total laparoscopic hysterectomy (TLH) is currently the standard procedure in patients requiring hysterectomy [1]. TLH, which is associated with less complication, shorter hospital stays, and faster return to normal activities, offers a safe and feasible alternative to abdominal hysterectomy [1]. Despite the benefits, laparoscopic hysterectomy has some limitations, such as a long learning curve and requirements of high-level surgical skills [2]. Robotic surgery could overcome these difficulties, making laparoscopic surgery possible for more patients [2]. Robotic surgery also enables surgeons to perform complex procedures more easily through improved visualization, more accurate instrument control, and easi- er use of instruments [3]. Gala et al. [3] found that robot-assisted hysterectomy was associated with a longer operation time but shorter length of hospital stay and less estimated blood loss (EBL) compared to laparoscopy and laparotomy.

Since the Food and Drug Administration approval of the da Vinci Robotic Surgical System for gynecologic surgery in 2005, robot-assisted surgery has become popular among both gynecologic surgeons and their patients [4]. Robotic surgical system offers a three-dimensional (3D) vision and EndoWrist instruments that mimic the human wrist and optimal ergonomics [2]. In Korea, the first robotic laparoscopic hysterectomy was performed by Kim et al. [5] in January 31st of 2006 after the approval by Korean FDA. In the gynecologic field, the da Vinci robotic surgical system is being
Correspondence to: Min Sun Kyung

Department of Obstetrics and Gynecology, Hallym University Dongtan Sacred Heart Hospital, Hallym University College of Medicine,

7 Keunjaebong-gil, Hwaseong 18450, Korea

Tel: +82-31-8086-2650, Fax: +82-31-8086-2029, E-mail: msfeel@hallym.or.kr

Received: May 21, 2018 / Accepted after revision: Jun. 2, 2018
(C) 2018 Soonchunhyang Medical Research Institute This is an Open Access article distributed under the terms of the Creative Commons Attribution Non-Commercial License (http://creativecommons.org/licenses/by-nc/4.0/). 
Robotic Single-Site Hysterectomy Might Be an Effective and Safe in Older and/or Obese Women with Large Uteri • Park YH, et al.

used in a wide range of specialties, including surgeries for uterine myoma, adenomyosis, endometrial hyperplasia, cervical intraepithelial neoplasia, and endometrial cancer $[5,6]$.

In robot-assisted surgery, each working port carries with it an inherent risk of bleeding, infection, concordant organ damage, hernia formation, and decreased cosmetic outcome [7,8]. Thus, the logical and natural goal in the field of minimally invasive surgery is to reduce the number of ports to perform the procedure $[7,8]$. Over the last years, the robotic single site system has been developed. This robotic single site system offers the automatic inversion of instruments, allowing more ample movements and better ergonomy when compared to non-robotic single-site laparoscopic surgery [9]. In addition to the 3D visualization, positioning of the surgeon on the robot console and the precise dissection of the anatomical structures result in a more accurate operation, with no collision of forceps [10]. The initial studies demonstrated that this technique is also safe and effective, and can help resolve the technical limitations found in laparoscopy $[11,12]$.

Although there are some argue that robotic single-site hysterectomy (RSSH) has several disadvantages, such as a longer operative time and higher cost, there have been few studies to compare surgical outcomes of RSSH and robot-assisted multiport hysterectomy (RH) [11].

We, therefore, retrospectively compared surgical and clinical outcomes of RSSH to those of $\mathrm{RH}$ for the treatment of benign uterine diseases in this study.

\section{MATERIALS AND METHODS}

From June 2015 to November 2017, total 38 patients underwent RSSH $(\mathrm{N}=12)$ and RH $(\mathrm{N}=26)$ for the treatment of benign uterine diseases at Hallym University Dongtan Sacred Heart Hospital. Medical records of all patients were retrospectively reviewed. This study was approved by the institutional review board of Hallym University Dongtan Sacred Heart Hospital (IRB approval no., 2018-02-007). Patients with confirmed gynecologic malignancies were excluded. All procedures were performed by two senior surgeons at the department of obstetrics and gynecology, Hallym University Dongtan Sacred Heart Hospital.

We performed $\mathrm{RH}$, regardless of the size of the uterus and suspicious pelvic adhesion, in all patients who want to undergo robotic surgery. RSSH was performed since the RSS system has been introduced to our hospital. The exclusion criteria for RSSH were immobile uteri with no descent on pelvic examination, uterine size greater than 20 gestational weeks, nulliparity, vagina narrower than two fingers wide, history of two or more previous abdominal surgeries, concomitant adnexal masses, and suspected uterine malignancy on ultrasound examination.

The two groups were compared for age, body mass index (BMI), parity, prior history of abdominal surgery, and comorbidities. The BMI was calculated according to the standard World Health Organization criteria. Vaginal delivery and cesarean section delivery were included in parity. Co-morbid medical diseases include hypertension, diabetes mellitus, hepatitis, tuberculosis, thyroid disease, acute transverse myelitis, and iron deficiency anemia. Surgical outcomes, including operation time (minutes), EBL (mL), uterus weight (g), hospital stay (day), preoperative hemoglobin $(\mathrm{Hb}$, $\mathrm{g} / \mathrm{dL}$ ), postoperative $\mathrm{Hb}$ change ( $\mathrm{g} / \mathrm{dL}$, change between preoperative $\mathrm{Hb}$ and postoperative one day and third day), postoperative transfusion, peri-operative complications, number of analgesics usage (times), and numeral rating scale (NRS) scores were compared between $\mathrm{RSSH}$ and $\mathrm{RH}$ groups. Patients were verbally rated their pain on a scale from 0 to 10 , with 0 equal to no pain and 10 equal to worst possible pain [13].

\section{Surgical techniques}

All surgeries were performed by experienced two senior surgeons. All patients underwent robot surgery using the da Vinci Xi surgical system (Intuitive Surgical, Sunnyvale, CA, USA).

\section{1) Robot-assisted multiport hysterectomy}

Under the general anesthesia, patients were placed in the dorsal lithotomy position with a gel pad underneath the patient for stabilization. The surgeon stood on the left side of the patient. The first assistant stood on the right side of the patient. The second assistant stood between the legs of the patient and modulated the RUMI uterine manipulator (Cooper Surgical Inc., Trumbull, CT, USA). RUMI uterine manipulator was placed with a $\mathrm{KOH}$ colpotomizer system in all patients. The optic port was $8 \mathrm{~mm}$ in diameter and was placed at the umbilicus or 3-5 $\mathrm{cm}$ above the umbilicus depending on the uterine size. An 8-mm robotic trocar was directly inserted at the umbilicus or 3-5 cm above the umbilicus. Pneumoperitoneum was induced by $\mathrm{CO}_{2}$ insufflations through umbilical trocar. Two robotic trocars, $8 \mathrm{~mm}$ each, were bilaterally $(8 \mathrm{~cm}$ distal to and level with the umbilicus) placed in the patient's abdomen. And an accessory 5-mm trocar was placed between the um- 

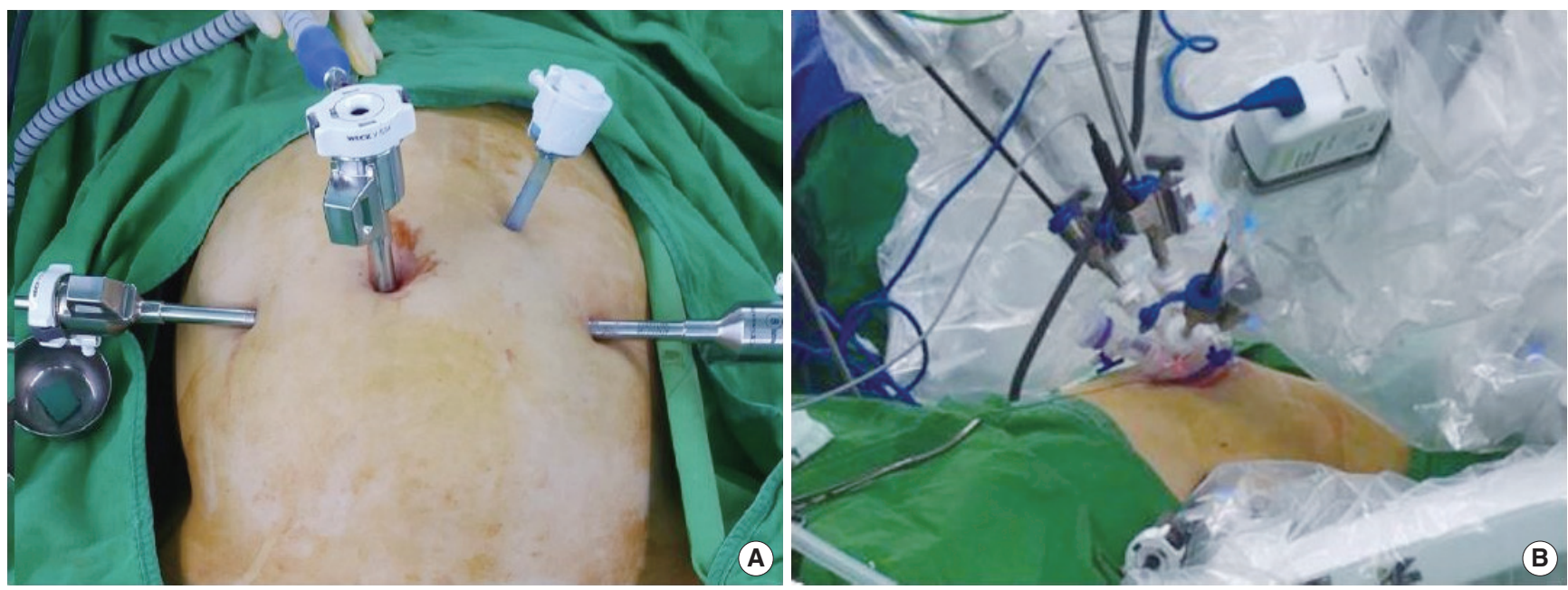

Fig. 1. Configuration of robot ports in robot-assisted multiport (A) and robotic single-site hysterectomy (B).

bilical and left lateral ports (Fig. 1A).

To facilitate surgery, the assistant used the accessory port to insert instruments (e.g., devices for retraction and suction/irrigation) and sutures. After the three-arm robotic tower was situated patient's right side, the middle arm was attached to the umbilical trocar for the laparoscope, and the two lateral arms were attached to the lateral trocars. The following robotic instruments were used: a fenestrated EndoWrist bipolar forceps on the left robotic arm and a monopolar EndoWrist curved scissors on the right side (Intuitive Surgical). All hysterectomies were performed in conventional manner. We started the hysterectomy by transection of the round ligament and then dissected of the broad ligament anteriorly and posteriorly. In premenopausal women we did not perform extra-adnexal hysterectomy. The bladder was dissected from the proximal vagina. The ascending branch of the uterine vessels were coagulated with bipolar current and transected by scissors. After transection of the cardinal ligaments, colpotomy was performed using the monopolar cutting current and the uterus was extracted vaginally. Large size uteri were cut into extractable parts with a knife and removed vaginally. The vaginal cuff was closed by robotic suturing using 1/0 V-Loc 180 Absorbable Wound Closure Device (Covidien, Mansfield, MA, USA), with continuous suture.

\section{2) Robotic single-site hysterectomy}

Under the general anesthesia, patients were placed in the dorsal lithotomy position with a gel pad underneath the patient for stabilization. RUMI uterine manipulator was placed with a $\mathrm{KOH}$ colpotomizer system in all patients. A single $2.5-\mathrm{cm}$ vertical umbili- cal incision was performed by an open Hasson approach to the level of fascia. The single-site multi-instrument silicon port (Lapsingle vision; Sejong Medical Co., Paju, Korea) was introduced through the fascial incision. After obtaining adequate pneumoperitoneum up to $14 \mathrm{~mm} \mathrm{Hg}$, the robotic column was docked between the patient's legs. An 8-mm 0-degree rigid laparoscope was introduced and watchful inspection of the total peritoneal cavity was completed. Subsequently, a 5×250 mm-curved cannula (arm 2) was introduced through the specific lumen, under constant visualization of the cannula tip by the camera. The same process was performed for the placement of arm 1. Finally, the specific robotic instruments were introduced (Fig. 1B): 5-mm fenestrated bipolar forceps on arm 2 and 5-mm monopolar hook on arm 1. The assistant kept exchanging the grasping forceps with the aspirator through a 5 to $10 \mathrm{~mm}$ passage in the same port. After all robotic instruments were situated, hysterectomies were performed in conventional manner. At the end of the hysterectomy, the surgical specimen was removed through the vagina. The vaginal cuff was closed by robotic suturing using 1/0 V-Loc 180 Absorbable Wound Closure Device (Covidien), with continuous suture.

At the end of the procedure, the robotic instruments and the camera were removed, and the robot was undocked. The umbilical incision was closed by layers, and the skin was done with intradermal sutures.

\section{Statistical methods}

All statistical analyses were performed using IBM SPSS ver. 24.0 (IBM Corp., Armonk, NY, USA). Dichotomous variables were 
Robotic Single-Site Hysterectomy Might Be an Effective and Safe in Older and/or Obese Women with Large Uteri • Park YH, et al.

Table 1. Patients' characteristics

\begin{tabular}{lcrc}
\hline Characteristic & $\begin{array}{c}\text { Robotic single-site } \\
\text { hysterectomy } \\
(\mathrm{N}=12)\end{array}$ & $\begin{array}{c}\text { Conventional } \\
(\mathrm{N}=26)\end{array}$ & P-value \\
\hline Age $(\mathrm{yr})$ & $49.5 \pm 5.05$ & $44.4 \pm 3.54$ & $0.001^{*}$ \\
Body mass index $\left(\mathrm{kg} / \mathrm{m}^{2}\right)$ & $27.4 \pm 2.47$ & $25.3 \pm 3.12$ & $0.045^{*}$ \\
Parity & $2.1 \pm 0.66$ & $1.7 \pm 0.68$ & 0.080 \\
No. of previous abdominal surgery & $0.5 \pm 0.79$ & $1.0 \pm 1.03$ & 0.121 \\
No. of co-morbid medical disease & $0.5 \pm 0.52$ & $0.2 \pm 0.43$ & 0.136 \\
\hline
\end{tabular}

Values are presented as mean \pm standard deviation.

${ }^{*} \mathrm{P}<0.05$.

Table 2. Surgical outcomes of RSSH and conventional robotic hysterectomy

\begin{tabular}{lccc}
\hline Surgical outcomes & $\mathrm{RSSH}(\mathrm{N}=12)$ & Conventional (N=26) & P-value \\
\hline Operative time (min) & $165.0 \pm 42.80$ & $159.2 \pm 48.82$ & 0.727 \\
Estimated blood loss (mL) & $115.8 \pm 33.15$ & $108.1 \pm 56.42$ & 0.662 \\
Uterus weight (g) & $445.9 \pm 157.21$ & $374.5 \pm 197.91$ & 0.291 \\
Hospital stay (day) & $5.4 \pm 0.51$ & $5.8 \pm 1.20$ & 0.289 \\
Preoperative Hb (g/dL) & $11.6 \pm 1.52$ & $11.5 \pm 1.86$ & 0.880 \\
Postoperative Hb change (g/dL) & $9.5 \pm 1.56$ & $9.7 \pm 1.38$ & 0.667 \\
Postoperative day 1 & $1.8 \pm 0.89$ & $1.4 \pm 1.53$ & 0.431 \\
Postoperative day 3 & $2.1 \pm 1.32$ & $1.7 \pm 1.83$ & 0.601 \\
Postoperative transfusion & & & 0.689 \\
$\quad$ No & $10(83.3)$ & $19(73.1)$ & \\
$\quad$ Yes & $2(16.7)$ & $2(7.7)$ & \\
Peri-operative complications & & & \\
Fever & $3(25.0)$ & $9(34.6)$ & 0.566 \\
Voiding difficulty & $1(8.3)$ & $1(3.8)$ & 0.577 \\
\hline
\end{tabular}

Values are presented as mean \pm standard deviation or number (\%).

$\mathrm{RSSH}$, robotic single-site hysterectomy; Hb, hemoglobin.

compared using the Fisher exact test or chi-square test. Continuous variables were compared using the t-test. Hazard ratio and $95 \%$ confidence interval were calculated. For all statistical tests, Pvalue less than 0.05 were considered significant.

\section{RESULTS}

Patients' characteristics were listed in Table 1. Parity, number of previous abdominal surgery, and co-morbid medical disease did not differ significantly between the two groups. Interestingly, women in RSSH group were significantly older ( $49.5 \pm 5.05$ years in RSSH vs. $44.4 \pm 3.54$ years in $\mathrm{RH}, \mathrm{P}=0.001$ ), and had significantly higher BMI $\left(27.4 \pm 2.47 \mathrm{~kg} / \mathrm{m}^{2}\right.$ in RSSH vs. $25.3 \pm 3.12 \mathrm{~kg} /$ $\mathrm{m}^{2}$ in $\left.\mathrm{RH}, \mathrm{P}=0.045\right)$ compared to those in $\mathrm{RH}$ group.

Surgical outcomes were described in Table 2. Operative time $(\mathrm{P}=0.727)$, EBL $(\mathrm{P}=0.662)$, uterus weight $(\mathrm{P}=0.291)$, postopera-
Table 3. Use of pain control and NRS score after RSSH and conventional robotic hysterectomy

\begin{tabular}{lccc}
\hline Variable & $\mathrm{RSSH}(\mathrm{N}=12)$ & Conventional $(\mathrm{N}=26)$ & P-value \\
\hline Additional analgesics & & & \\
Postoperative $6 \mathrm{hr}$ & $3(25.0)$ & $2(7.7)$ & 0.689 \\
Postoperative $24 \mathrm{hr}$ & $1(8.3)$ & $4(15.4)$ & 0.120 \\
Postoperative $48 \mathrm{hr}$ & $1(8.3)$ & $4(15.4)$ & 0.131 \\
NRS score & & & \\
Postoperative $6 \mathrm{hr}$ & $1.6 \pm 2.23$ & $2.9 \pm 2.05$ & 0.095 \\
Postoperative $24 \mathrm{hr}$ & $0.5 \pm 1.17$ & $0.6 \pm 0.70$ & 0.802 \\
Postoperative $48 \mathrm{hr}$ & $0.3 \pm 0.89$ & $0.2 \pm 0.37$ & 0.380 \\
\hline
\end{tabular}

Values are presented as mean \pm standard deviation or number $(\%)$.

NRS, numeral rating scale; RSSH, robotic single-site hysterectomy.

tive hospital stay $(\mathrm{P}=0.289)$, postoperative $\mathrm{Hb}$ change in day 1 $(\mathrm{P}=0.431)$, and day $3(\mathrm{P}=0.601)$ did not significantly differ between the two groups.

No significant differences could be found in perioperative complications. There were 14 minor complications: four in the RSSH group and 10 in the $\mathrm{RH}$ group. In RSSH group, there were three patients who had fever after operation and one patient who had voiding difficulty after removal of Foley catheter, but all patients were discharged after recovery. In RH group, nine patients had fever after operation and one had voiding difficulty after removal of Foley catheter, but all patients were discharged after recovery (Table 2).

We compared the use of additional analgesics and postoperative NRS pain score between the two groups (Table 3 ). The rate of use for additional analgesics at postoperative 6 hours $(P=0.689), 24$ hours $(\mathrm{P}=0.120)$, and 48 hours $(\mathrm{P}=0.131)$ were not different between the two groups. Also, NRS score at postoperative 6 hours $(\mathrm{P}=0.095), 24$ hours $(\mathrm{P}=0.802)$, and 48 hours $(\mathrm{P}=0.380)$ were not different between the two groups.

\section{DISCUSSION}

According to our data, the surgical outcomes and postoperative pain of RSSH were comparable to those of $\mathrm{RH}$, in spite of the older mean age and greater BMI of women in RSSH group compared to RH.

Since 2005, robotic surgery has been widely accepted and performed for the treatment of benign and malignant gynecologic disease [14]. The robotic surgery provides better visualization of surgical field with 3D- and wrist-like movements with robotic arms, which results in less postoperative pain and severe compli- 
Park YH, et al. • Robotic Single-Site Hysterectomy Might Be an Effective and Safe in Older and/or Obese Women with Large Uteri

cations, such as hemorrhage and adjacent organ injury [14]. However, the port site incision in robotic surgery is larger than conventional laparoscopy and also the number of ports required during the procedure is greater than conventional laparoscopy. To minimize the number of scar and provide better cosmetic results, single-port access laparoscopic surgery has been developed and widely performed for the treatment of benign gynecologic disease [15].

Similar to the laparoscopic surgery, single-site robotic surgery has also been developed [15-17]. Increasing the application of single-site robotic surgery has been described through several case reports and case series $[15,18,19]$. Those reports suggested that the single-site robotic surgery is safe and feasible technique for the surgical treatment of benign and malignant diseases expanded within general, urological, gynecological, and pediatric surgery [15,18-20]. A case series report of the robotic single-site pyeloplasty revealed that it is safely performed for selected patients, such as younger patients ( $<45$ years) with no prior abdominal surgery [18]. The authors suggested that the key techniques for the successful procedure were staggering, a "chopstick" arrangement of the instruments, and use of a $30^{\circ}$ lens in an upward configuration [18].

There have been a number of reports comparing single-site robotic surgery to conventional multi-port laparoscopy [21-23]. A matched-cohort study comparing 16 single-port robotic-assisted adrenalectomies with 16 patients from a pool of 148 laparoscopic adrenalectomies revealed that surgical outcomes, including operative time, severe complication, and hospital stay were not different between the two groups [22]. In addition, patients in single-port robotic-assisted adrenalectomy group used less narcotic pain medication in the first 24 hours after surgery compared to those in laparoscopic adrenalectomy group (43 mg vs. $84 \mathrm{mg}, \mathrm{P}<0.001$ ) [22].

Another retrospective case-control study comparing robotic single-site surgery and conventional laparoscopy revealed that clinical outcomes of robotic single-site surgery were comparable to those of conventional laparoscopy in selected patients [21].

There have been few studies comparing the RH to RSSH techniques in gynecologic surgery [24,25]. In a retrospective study comparing clinical characteristics, surgical outcomes, and cost of RSSH $(\mathrm{N}=45)$ and $\mathrm{RH}(\mathrm{N}=59)$, there was no significant difference in console time, surgical complication rate, conversion rate, and postoperative pain between the two groups [25]. Although the docking time of RSSH group was significantly longer than $\mathrm{RH}$ group $(\mathrm{P}=0.001)$, EBL was less and hospital stay was shorter in the
RSSH group ( $\mathrm{P}<0.001$ and $\mathrm{P}=0.009$, respectively) [25]. In addition, the cost analysis showed significant differences in favor of RSSH [25].

Similarly, in our data, surgical outcomes including operative time, EBL and peri-operative complications did not significantly differ between the two groups.

As for the postoperative pain, RSSH appears to be associated with a similar or less pain and analgesic use. In our data, the additional use of pain control at postoperative 6 hours in RSSH group was less than that in RH group (1.6 vs. 2.9), although the differences were not statistically significant $(\mathrm{P}=0.095)$. A prior study comparing postoperative pain and analgesic drugs after $\mathrm{RH}$ and $\mathrm{RSSH}$ reported that RSSH was associated with a better control of postoperative pain and postoperative analgesic drugs [25]. Postoperative pain at 12 hours was similar between the two groups $(\mathrm{P}=0.870)$, whereas it was significantly lower in the RSSH group than in the RH group at 24 hours $(\mathrm{P}=0.009)$ [25].

Although RSSH shows comparable surgical outcomes and postoperative pain to $\mathrm{RH}$, surgical indication of single-site laparoscopic surgery is considered to be relatively narrow $[9,26]$. Specifically, a large uterus (more than 16-week gestation size) is thought to be a potential contraindication of single-site laparoscopic surgery $[27,28]$. However, the development of surgical skill and devices makes it possible to remove large uterus using robotic single-site surgery [28]. A systematic review revealed that the weight of the removed uteri by RSSH ranged from 39 to $520 \mathrm{~g}$ [28]. In our data, the mean uterine weight in the RSSH and $\mathrm{RH}$ was $445.9 \mathrm{~g}$ and $374.5 \mathrm{~g}$, respectively. The mean uterine size of patients enrolled in our study was large compared with other studies [25-28]. In spite of the large uterine size, all cases were successfully completed RSSH without conversion to RH or laparotomy. We consider that adequate patient selection for the RSSH was the key to the successful procedure.

Compared with other hospitals, our hospital stay is little longer than others it is because of our hospital clinical pathway of 5 days. The patient admitted the day before surgery and discharged on the third day after surgery.

In our data, the mean age was older and BMI was greater in $\mathrm{RSSH}$ group compared to RH group. Generally, it is considered to be safe to perform robotic single-site surgery in younger patients [18]. In addition, obesity is considered to be a relative contraindication for single-site laparoscopic surgery [29]. A prospective study including 78 patients undergoing robotic single-site adrenalecto- 
my revealed that the higher BMI was associated with a longer operative time [29]. In spite of older age and higher BMI, which can make it difficult to obtain adequate surgical field during the procedure, all RSSH was successfully completed with comparable surgical outcomes to RH. With this, we could hypothesize that $\mathrm{RSSH}$ is feasible and safe even in older and/or obese patients.

Our study has some limitations, such as a retrospective study and small number of study and control group. In addition, we did not divided detailed operating time such as port placement time, docking time, console time, time for hysterectomy, and time for vaginal cuff closure. In spite of those limitations, our data is valuable to prove surgical feasibility and safety of RSSH compared to $\mathrm{RH}$. The strengths of our study stem from the homogeneity of surgical procedures performed by two senior surgeons with welltrained surgical team.

In conclusion, $\mathrm{RSSH}$ is feasible and equally safe with RH even in patients with large uteri, older age, and higher BMI.

\section{REFERENCES}

1. Nieboer TE, Johnson N, Lethaby A, Tavender E, Curr E, Garry R, et al. Surgical approach to hysterectomy for benign gynaecological disease. Cochrane Database Syst Rev 2009;(3):CD003677.

2. Van Weelden WJ, Gordon BBM, Roovers EA, Kraayenbrink AA, Aalders CIM, Hartog F, et al. Perioperative surgical outcome of conventional and robot-assisted total laparoscopic hysterectomy. Gynecol Surg 2017;14:5.

3. Gala RB, Margulies R, Steinberg A, Murphy M, Lukban J, Jeppson P, et al. Systematic review of robotic surgery in gynecology: robotic techniques compared with laparoscopy and laparotomy. J Minim Invasive Gynecol 2014;21:353-61.

4. Madueke-Laveaux OS, Advincula AP. Robot-assisted laparoscopy in benign gynecology: advantageous device or controversial gimmick? Best Pract Res Clin Obstet Gynaecol 2017;45:2-6.

5. Kim YT, Kim SW, Jung YW. Robotic surgery in gynecologic field. Yonsei Med J 2008;49:886-90.

6. Jung YW, Kim YT, Lee DW, Hwang YI, Nam EJ, Kim JH, et al. The feasibility of scarless single-port transumbilical total laparoscopic hysterectomy: initial clinical experience. Surg Endosc 2010;24:1686-92.

7. Kommu SS, Kaouk JH, Rane A. Laparo-endoscopic single-site surgery: preliminary advances in renal surgery. BJU Int 2009;103:1034-7.

8. Escobar PF, Starks D, Fader AN, Catenacci M, Falcone T. Laparoendoscopic single-site and natural orifice surgery in gynecology. Fertil Steril 2010;94:2497-502.

9. Gomes MTV, Machado AMN, Podgaec S, Barison GAS. Initial experience with single-port robotic hysterectomy. Einstein (Sao Paulo) 2017;15:47680.

10. Uppal S, Frumovitz M, Escobar P, Ramirez PT. Laparoendoscopic singlesite surgery in gynecology: review of literature and available technology. J Minim Invasive Gynecol 2011;18:12-23.

11. Geetha P, Nair MK. Laparoscopic, robotic and open method of radical hysterectomy for cervical cancer: a systematic review. J Minim Access Surg
2012;8:67-73

12. Escobar PF, Knight J, Rao S, Weinberg L. Da Vinci ${ }^{\circledR}$ single-site platform: anthropometrical, docking and suturing considerations for hysterectomy in the cadaver model. Int J Med Robot 2012;8:191-5.

13. Bijur PE, Latimer CT, Gallagher EJ. Validation of a verbally administered numerical rating scale of acute pain for use in the emergency department. Acad Emerg Med 2003;10:390-2.

14. Advincula AP, Wang K. Evolving role and current state of robotics in minimally invasive gynecologic surgery. J Minim Invasive Gynecol 2009;16: 291-301.

15. Kirshtein B, Haas EM. Single port laparoscopic surgery: concept and controversies of new technique. Minim Invasive Surg 2012;2012:456541.

16. Cela V, Freschi L, Simi G, Ruggiero M, Tana R, Pluchino N. Robotic single-site hysterectomy: feasibility, learning curve and surgical outcome. Surg Endosc 2013;27:2638-43.

17. Bogliolo S, Mereu L, Cassani C, Gardella B, Zanellini F, Dominoni M, et al. Robotic single-site hysterectomy: two institutions' preliminary experience. Int J Med Robot 2015;11:159-65.

18. Tobis S, Houman J, Thomer M, Rashid H, Wu G. Robot-assisted transumbilical laparoendoscopic single-site pyeloplasty: technique and perioperative outcomes from a single institution. J Laparoendosc Adv Surg Tech A 2013;23:702-6.

19. Angus AA, Sahi SL, McIntosh BB. Learning curve and early clinical outcomes for a robotic surgery novice performing robotic single site cholecystectomy. Int J Med Robot 2014;10:203-7.

20. Bosi HR, Guimaraes JR, Cavazzola LT. Robotic assisted single site for bilateral inguinal hernia repair. Arq Bras Cir Dig 2016;29:109-11.

21. El Hachem L, Andikyan V, Mathews S, Friedman K, Poeran J, Shieh K, et al. Robotic single-site and conventional laparoscopic surgery in gynecology: clinical outcomes and cost analysis of a matched case-control study. J Minim Invasive Gynecol 2016;23:760-8.

22. Arghami A, Dy BM, Bingener J, Osborn J, Richards ML. Single-port robotic-assisted adrenalectomy: feasibility, safety, and cost-effectiveness. JSLS 2015;19:e2014.00218.

23. Vyas D, Weiner C, Vyas AK. Current status of single-site robotic cholecystectomy, its feasibility, economic and overall impact. Am J Robot Surg 2014;1:1-64.

24. Moukarzel LA, Sinno AK, Fader AN, Tanner EJ. Comparing single-site and multiport robotic hysterectomy with sentinel lymph node mapping for endometrial cancer: surgical outcomes and cost analysis. J Minim Invasive Gynecol 2017;24:977-83.

25. Bogliolo S, Ferrero S, Cassani C, Musacchi V, Zanellini F, Dominoni M, et al. Single-site versus multiport robotic hysterectomy in benign gynecologic diseases: a retrospective evaluation of surgical outcomes and cost analysis. J Minim Invasive Gynecol 2016;23:603-9.

26. Paek J, Lee JD, Kong TW, Chang SJ, Ryu HS. Robotic single-site versus laparoendoscopic single-site hysterectomy: a propensity score matching study. Surg Endosc 2016;30:1043-50.

27. Phongnarisorn C, Chinthakanan O. Transumbilical single-incision laparoscopic hysterectomy with conventional laparoscopic instruments in patients with symptomatic leiomyoma and/or adenomyosis. Arch Gynecol Obstet 2011;284:893-900.

28. Iavazzo C, Minis EE, Gkegkes ID. Single-site port robotic-assisted hysterectomy: an update. J Robot Surg 2018;12:201-13.

29. Tsai YC, Chen CH, Hu YH, Er LK, Wu CH, Chueh SC, et al. Factors affecting operative efficiency and post-operative convalescence in laparoendoscopic single-site (LESS) adrenalectomy. Surg Endosc 2018;32:144955 . 